\title{
Teoría Bifásica del Movimiento Dentario Aplicada Mediante Micro-Osteo-Perforaciones
}

\author{
Teeth Movement Bifasic Theory Applied by Micro-Osteo-Perforations
}

\author{
Carolina Minte Hidalgo'; Mani Alikhani²; Cristina Teixeira ${ }^{3} \&$ Paulo Sandoval Vidal ${ }^{4}$
}

MINTE, H. C.; ALIKHANI, M.; TEIXEIRA, C. \& SANDOVAL, V. P. Teoría bifásica del movimiento dentario aplicada mediante micro-osteo-perforaciones. Int. J. Odontostomat., 13(2):180-183, 2019.

RESUMEN: Un desafío común en la ortodoncia es la realización de cierres de espacio en brechas largas con pérdida ósea significativa producto de extracciones tempranas, sitio de extracciones traumáticas o expansiones en adultos con tabla vestibular delgada. El propósito de este artículo es difundir una teoría del movimiento dental desarrollada a partir de una serie de investigaciones que intentan probarla en animales como seres humanos. Las dos fases de remodelación de hueso son la "activación - reabsorción" (proceso catabólico) y "activación-formación" (proceso anabólico) de las superficies del hueso, resultando en los cambios de tamaño, forma y posición del hueso. La inflamación es necesaria para el movimiento dentario. Se puede estimular ambas fases mediante pequeñas perforaciones del hueso que pueden ser realizadas de forma segura en la superficie vestibular o lingual de las tablas corticales pudiendo ser superficiales o profundas. Se muestran ejemplos clínicos de pacientes tratados con el enfoque de la Teoría bifásica mediante estimulación ósea transgingival. Se concluye que esta teoría bifásica permite explicar la favorable respuesta que se observa en situaciones clínicas complejas cuando se estimula el movimiento con micro-osteoperforaciones.

PALABRAS CLAVE: técnicas de movimiento dental, ortodoncia, ortopedia, procedimientos quirúrgicos sin sutura, resorción ósea.

\section{INTRODUCCIÓN}

El movimiento dental en ortodoncia, producido por la aplicación de fuerzas mecánicas, es el resultado de una actividad dinámica de aposición y reabsorción osea (O'Connor et al., 1982). La modelación ósea y su remodelación es determinante para el movimiento dental. Tres tipos de células óseas juegan un rol significativo en la biología del movimiento dentario: osteoblastos, osteocitos y osteoclastos (Alikhani, 2017).

Las fuerzas ortodóncicas producen diferentes tipos de movimiento dependiendo de la magnitud y dirección de la fuerza aplicada a los dientes. Cada tipo de movimiento dentario es causado por un estrés específico distribuido alrededor del ligamento periodontal y hueso alveolar. Es extensamente aceptado que las áreas que experimentan el mayor estrés compresivo presentan los mayores niveles de reabsorción ósea osteoclástica. Muchas teorías han sido propuestas para explicar el evento inicial que conduce a la activación osteoclástica en los sitios de compresión (Domínguez \& Velásquez, 2017). En general estas teorías se dividen en 2 áreas: una propone que las células óseas (específicamente los osteocitos) son el objetivo directo de las fuerzas ortodóncicas, la otra propone que es el ligamento periodontal. En ambas teorías son los osteoclastos los que reabsorben el hueso, por lo tanto, serían las células que controlan el grado de movimiento dental (Henneman et al., 2008). Basados en la primera teoría (directa), se propone la existencia de dos mecanismos por los que se produce la activación de los osteocitos. El primero, cuando la estimulación mecánica está en niveles fisiológicos, los osteocitos miden los diferentes componentes de la

\footnotetext{
${ }^{1}$ Alumna del Programa de Magister en Odontología, Universidad de La Frontera, Temuco, Chile.

2 Departamento de Biología del desarrollo y Ortodoncia, Facultad de Medicina Dental de Harvard, Boston, USA.

${ }^{3}$ Profesora Asociada de Ortodoncia, ciencias básicas y biología craneofacial, Departamento de Ortodoncia de la Facultad de Odontología de la Universidad de Nueva York, USA.

${ }^{4}$ Facultad de Odontología, Universidad de La Frontera, Chile.
} 
estimulación mecánica (como la deformación de la matriz) y dirigen la maquinaria de remodelación ósea para que los osteoclastos remuevan el hueso antiguo y se produzca reconstrucción ósea por activación osteoblástica. Por éste mecanismo, el movimiento ortodóncico es considerado una adaptación fisiológica a la estimulación mecánica inducida por fuerzas ortodóncicas. El segundo mecanismo señala que cuando existen cargas mecánicas más elevadas (patológicas) se producen microfracturas en la matriz, las que son detectadas por los osteocitos, resultando en un incremento de la remodelación ósea en el sitio afectado. Por este mecanismo, el movimiento ortodóncico es considerado una respuesta al trauma causado por las fuerzas ortodoncicas (Henneman et al.). La respuesta fisiológica y patológica a las fuerzas ortodóncicas pueden tener diferentes orígenes, pero inicialmente ambas producen una respuesta inflamatoria aséptica aguda, con una temprana liberación de quimiocinas por parte de las células locales. Estas son pequeñas proteínas que son capaces de atraer células distantes a la zona, facilitando la expresión de moléculas de adhesión en los vasos sanguíneos y estimulando el reclutamiento de células inflamatorias y precursoras desde la microvasculatura hacia el espacio extravascular (Taddei et al., 2012).

Poco tiempo después de aplicar fuerzas ortodóncicas, un gran espectro de mediadores son liberados por células inflamatorias y locales. Estos mediadores inflamatorios son las citoquinas, proteínas extracelulares que inducen tanto procesos antiinflamatorios, como procesos proinflamatorios (Garlet et al., 2007; Teixeira et al., 2010). Otras dos clases de mediadores inflamatorios que son liberados durante el movimiento ortodóncico son las prostaglandinas y los neuropéptidos. Las prostaglandinas incrementan la permeabilidad vascular y la adhesión celular (Ricciotti \& Fitzgerald, 2011). Los neuropéptidos son pequeñas proteínas que transmiten señales de dolor, regulan el tono y modulan la permeabilidad vascular (Lundy \& Linden, 2004). Es así como para amplificar las reacciones corporales a las fuerzas ortodóncicas se puede intentar incrementar la liberación de citoquinas (si se piensa que la respuesta inflamatoria del ligamento periodontal y del hueso son la clave para controlar el grado de movimiento dentario) u optimizar la estimulación mecánica (si se cree que el movimiento dentario es una respuesta fisiológica directa a la estimulación mecánica). Estos enfoques incluyen una inducción local o sistémica de diferentes factores químicos o aplicación de estimulación física que pueda incrementar el número de osteoclastos independiente de la fuerza ortodóncica. Es así como el fenómeno biológico del movimiento dentario es el resultado de respuestas a las fuerzas ortodóncicas fuertemente acopladas entre osteocitos, osteoclastos y osteoblastos. La evidencia apunta a la conversión de fuerzas ortodóncicas en una secuencia de catabolismo temporal seguido de anabolismo en el hueso alveolar (Cheung et al., 2016).

\section{Una nueva teoría}

Tomando todos estos datos sobre el movimiento dentario es que se logró desarrollar la Teoría Bifásica del movimiento dentario, para explicar no sólo las consecuencias biológicas del tratamiento de ortodoncia, sino que también para guiar un tratamiento de ortodoncia acelerado, eficaz y seguro (Alikhani). En la Teoría Bifásica del movimiento dentario, los osteoclastos juegan un rol importante en la activación de los osteoblastos. En ella se concuerda con varios estudios que sugieren que los osteoclastos son los principales reguladores de los osteoblastos (Matsuo \& Irie, 2008). Este efecto puede ocurrir a través de tres vías: (1) los osteoclastos lanzan factores paracrinos que directamente reclutan y activan osteoblastos; (2) los osteoclastos activan a los osteoblastos a través de una interacción directa célula-célula; (3) la reabsorción ósea por osteoclastos expone proteínas de la matriz ósea, las que indirectamente atraen y activan los osteoblastos. En todos los casos, la actividad osteoclástica precede la actividad osteoblástica (Chou \& Alikhani, 2017).

La creciente demanda por tratamientos ortodóncicos rápidos, especialmente en pacientes adultos, ha dirigido el foco de las investigaciones en acelerar el movimiento dentario reduciendo la duración del tratamiento, pero manteniendo su eficacia.

Hasta la fecha, varios modelos de movimiento ortodóncico acelerado han sido reportados, entre ellos, farmacoterapia (Hashimoto et al., 2001), terapia de láser de baja intensidad (Doshi-Mehta \& Bhad-Patil, 2012), campos de pulso electromagnéticos (Stark \& Sinclair, 1987), corriente eléctrica (Davidovitch et al., 1980), corticotomía (Peron et al., 2017), la vibración mecánica (Alikhani et al., 2016, 2018), la piezoincisión (Aksakalli et al., 2016) y las micro-osteoperforaciones (Alikhani et al., 2015). Estas últimas presentan un enfoque innovador, menos invasivo y más eficiente. Consiste en pequeñas y superficiales osteoperforaciones que pueden ser colocados de forma segura en la superficie vestibular o lingual de las tablas corticales por 
el ortodoncista tratante, con efectos secundarios leves y dolor o incomodidad limitados (Alikhani et al., 2013). Tanto en estudios en animales como en humanos, la aplicación de osteoperforaciones poco profundas en la proximidad del diente en movimiento resulta en un incremento significativo de inflamación, activación osteoclástica, remodelación ósea y movimiento dentario (Texeira et al., 2010). Las micro-osteoperforaciones pueden estimular una respuesta inflamatoria más grande de dos modos: 1) incrementando el número de perforaciones o 2) incrementando la profundidad de penetración de estas. En los casos en los que se requiere el efecto catabólico y anabólico de las microosteoperforaciones, como lo es en la movilización dentaria en brechas largas con pérdida ósea, la combinación de unas pocas perforaciones profundas de 3-7 mm (efecto catabólico) con múltiples perforaciones superficiales de 1-2 mm (efecto anabólico) estimulará ambos procesos, con una frecuencia de aplicación de las perforaciones cada 28-56 días (Alikhani).

\section{Proyecciones clínicas}

Uno de los desafíos de los tratamientos ortodóncicos es movilizar dientes en áreas con significativa pérdida ósea, como lo son: crestas alveolares residuales de extracciones antiguas, zonas con falta de desarrollo de hueso alveolar por presencia de dientes anquilosados, sitio de extracciones traumáticas, expansiones en adultos con tabla vestibular delgada o pobre base ósea.

Es posible realizar las micro-osteoperforaciones con la punta afilada de instrumentos manuales, como agujas u otros quirúrgicos. También es posible hacerlo con microtornillos, sin embargo se debe contar con un mango que permita tomar firmemente el tornillo ya que debe introducirse y luego retirarse de manera segura (Fig. 1). Para facilitar la aplicación de las microosteoperforaciones se ha desarrollado una herramienta que permite a los clínicos establecer una longitud determinada para la punta de corte, lo que permite que sea un procedimiento seguro y predecible acorde con la evaluación clínica del espesor de soporte periodontal de cada paciente (Fig. 2). Las herramientas están disponibles tanto en forma manual como de baja velocidad, en caso de que el hueso cortical denso no permita una penetración adecuada.

También se ha probado con otros elementos que injurien el hueso de manera controlada, como es con una fresa de tungsteno de $0,8 \mathrm{~mm}$ (Fig. 3) con una pieza de mano de bajo torque.

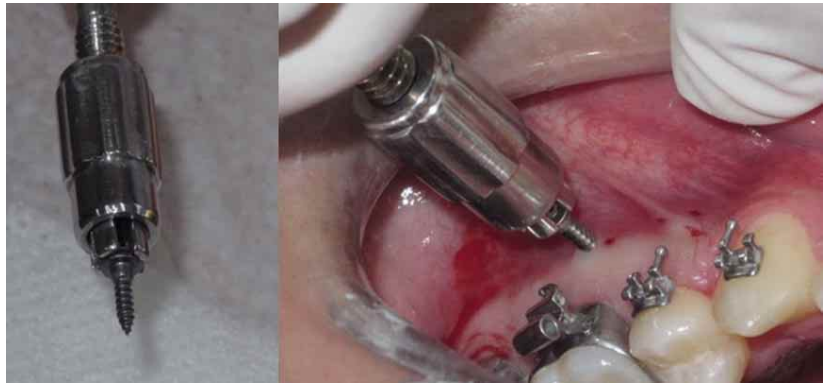

Fig. 1. Micro-osteoperforaciones realizadas con microtornillos de titanio en foma manual.

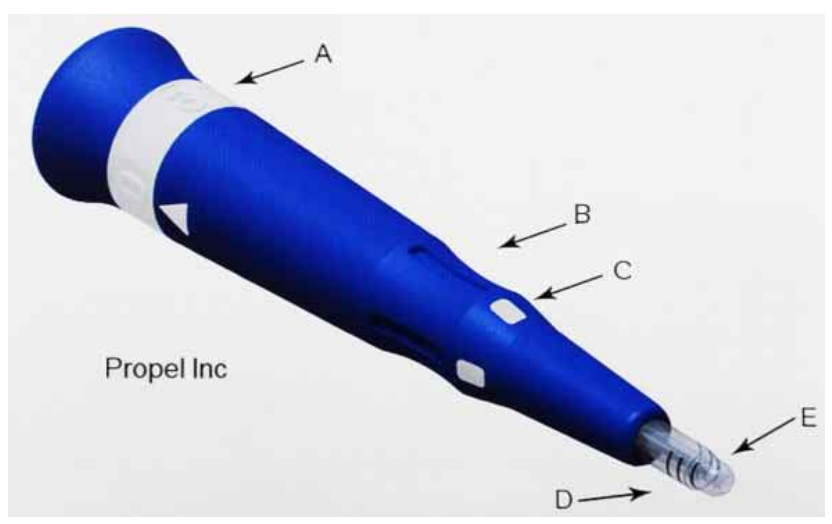

Fig. 2. Micro-osteoperforador mecánico motorizado de Propel Inc. (A) Dial para ajustar la profundidad entre 3 y $7 \mathrm{~mm}$; (B) Posicionador del dedo índice; (C) LED indicador de detención de acuerdo con la profundidad; (D) Punta; (E) Cubierta retráctil.

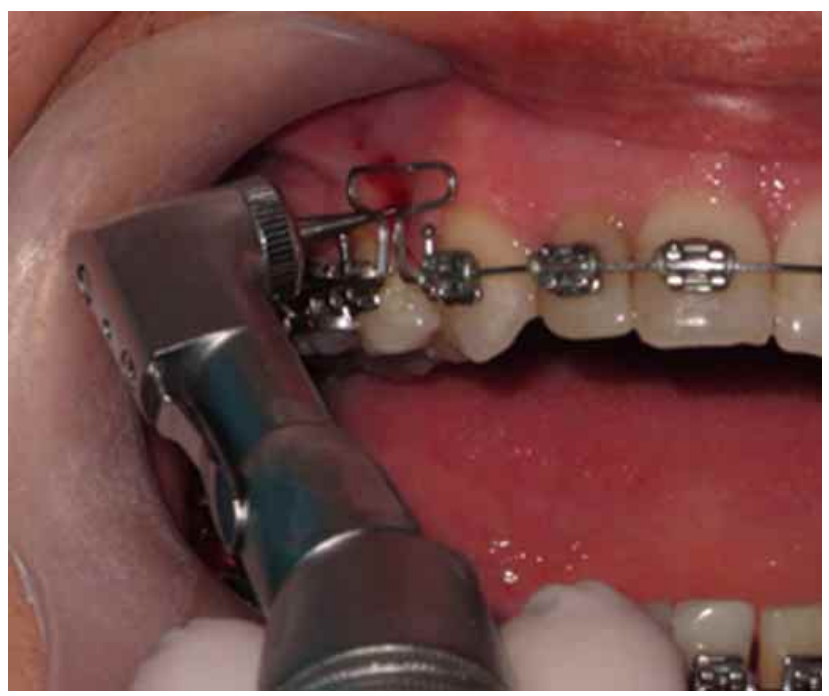

Fig. 3. Micro-osteoperforaciones realizada con micromotor con reducción de torque de 1:16.000 revoluciones.

CONCLUSIONES. La teoría bifásica del movimiento dentario permite explicar las diferentes respuestas que se observan clínicamente como respuesta a las fuerzas ortodóncicas cuando se estimula con microosteoperforaciones en diferentes profundidades a través de la encía adherida. 
MINTE, H. C.; ALIKHANI, M.; TEIXEIRA, C. \& SANDOVAL, V. P. Teeth movement bifasic theory applied by micro-osteoperforations. Int. J. Odontostomat., 13(2):180-183, 2019.

ABSTRACT: A common challenge in orthodontics is the task of space closures in long gaps with significant bone loss due to early extractions, site of traumatic extractions or expansions in adults with thin vestibular table. The purpose of this article is to disseminate a theory of dental movement developed from a series of investigations that try to test it in animals as human beings. The two phases of bone remodeling are the "activation - resorption" (catabolic process) and "activation-formation" (anabolic process) of bone surfaces, resulting in changes in bone size, shape and position. Inflammation is necessary for tooth movement. Both phases can be stimulated by small perforations of the bone that can be performed safely on the vestibular or lingual surface of the cortical boards, which may be superficial or deep. Clinical examples of patients treated with the biphasic theory approach by transgingival bone stimulation are shown. It is concluded that this biphasic theory allows to explain the favorable response observed in complex clinical situations when the movement is stimulated with micro-osteoperforations.

KEY WORDS: tooth movement techniques, orthodontics, orthopedics, sutureless surgical procedures, bone resorption.

\section{REFERENCIAS BIBLIOGRÁFICAS}

Aksakalli, S.; Calik, B.; Kara, B. \& Ezirganli, S. Accelerated tooth movement with piezocision and its periodontal-transversal effects in patients with Class II malocclusion. Angle Orthod., 86(1):59-65, 2016.

Alikhani, M. Clinical Guide to Accelerated Orthodontics: Whit a Focus of Micro-Osteoperforations. Cham, Springer International Publishing AG, 2017.

Alikhani, M.; Alansari, S.; Hamidaddin, M. A.; Sangsuwon, C.; Alyami, B.; Thirumoorthy, S. N.; Oliveira, S. M.; Nervina, J. M. \& Teixeira, C. C. Vibration paradox in orthodontics: Anabolic and catabolic effects. PLoS One, 13(5):e0196540, 2018.

Alikhani, M.; Alansari, S.; Sangsuwon, C.; Alikhani, M.; Chou, M. Y.; Alyami, B.; Nervina, J. M. \& Teixeira, C. C. Micro-osteoperforations: Minimally invasive accelerated tooth movement. Semin. Orthod., 21(3):162-9, 2015.

Alikhani, M.; Lopez, J. A.; Alabdullah, H.; Vongthongleur, T.; Sangsuwon, C.; Alikhani, M.; Alansari, S.; Oliveira, S. M.; Nervina, J. M. \& Teixeira, C. C. High-frequency acceleration: therapeutic tool to preserve bone following tooth extractions. J. Dent. Res., 95(3):311-8, 2016.

Alikhani, M.; Raptis, M.; Zoldan, B.; Sangsuwon, C.; Lee, Y. B.; Alyami, B.; Corpodian, C.; Barrera, L. M.; Alansari, S.; Khoo, E. \& Teixeira, C. Effect of micro-osteoperforations on the rate of tooth movement. Am. J. Orthod. Dentofacial Orthop., 144(5):639-48, 2013.

Cheung, T.; Park, J.; Lee, D.; Kim, C.; Olson, J.; Javadi, S.; Lawson, G.; McCabe, J.; Moon, W.; Ting, K.; et al. Ability of mini-implantfacilitated micro-osteoperforations to accelerate tooth movement in rats. Am. J. Orthod. Dentofacial Orthop., 150(6):958-67, 2016.

Chou, M. Y. \& Alikhani, M. A successful story of translational orthodontic research: Micro-osteoperforation-from experiments to clinical practic. APOS Trends Orthod., 7(1):6-11, 2017.
Davidovitch, Z.; Finkelson, M. D.; Steigman, S.; Shanfeld, J. L.; Montgomery, P. C. \& Korostoff, E. Electric currents, bone remodeling, and orthodontic tooth movement. II. Electric currents, bone remodeling, and orthodontic tooth movement. II. Increase in rate of tooth movement and periodontal cyclic nucleotide levels by combined force and electric current. Am. J. Orthod., 77(1):33-47, 1980.

Domínguez, C. \& Velásquez, C. Reevaluation of the Strain Pressure Theory. Toward a better understanding the biology of tooth movement. Int. J. Odontostomat., 11(2):133-40, 2017.

Doshi-Mehta, G. \& Bhad-Patil, W. A. Efficacy of low-intensity laser therapy in reducing treatment time and orthodontic pain: a clinical investigation. Am. J. Orthod. Dentofacial Orthop., 141(3):289-97, 2012.

Garlet, T. P.; Coelho, U.; Silva, J. S. \& Garlet, G. P. Cytokine expression pattern in compression and tension sides of the periodontal ligament during orthodontic tooth movement in humans. Eur. J. Oral Sci., 115(5):355-62, 2007.

Hashimoto, F.; Kobayashi, Y.; Mataki, S.; Kobayashi, K.; Kato, Y. \& Sakai, $\mathrm{H}$. Administration of osteocalcin accelerates orthodontic tooth movement induced by a closed coil spring in rats. Eur. J. Orthod., 23(5):535-45, 2001.

Henneman, S.; Von den Hoff, J. W. \& Maltha, J. C. Mechanobiology of tooth movement. Eur. J. Orthod., 30(3):299-306, 2008.

Lundy, F. T. \& Linden, G. J. Neuropeptides and neurogenic mechanisms in oral and periodontal inflammation. Crit. Rev. Oral Biol. Med., 15(2):82-98, 2004.

Matsuo, K. \& Irie, N. Osteoclast-osteoblast communication. Arch. Biochem. Biophys., 473(2):201-9, 2008.

O'Connor, J. A.; Lanyon, L. E. \& MacFie, H. The influence of strain rate on adaptive bone remodelling. J. Biomech., 15(10):767-81, 1982.

Peron, A. P.; Johann, A. C.; Papalexiou, V.; Tanaka, O. M.; GuarizaFilho, O.; Ignácio, S. A. \& Camargo, E. S. Tissue responses resulting from tooth movement surgically assisted by corticotomy and corticision in rats. Angle Orthod., 87(1):118-24, 2017.

Ricciotti, E. \& Fitzgerald, G. A. Prostaglandins and inflammation. Arterioscler. Thromb. Vasc. Biol., 31(5):986-1000, 2011.

Stark, T. M. \& Sinclair, P. M. Effect of pulsed electromagnetic fields on orthodontic tooth movement. Am. J. Orthod. Dentofacial Orthop. 91(2):91-104, 1987.

Taddei, S. R.; Andrade, I. Jr.; Queiroz-Junior, C. M.; Garlet, T. P.; Garlet, G. P.; Cunha, F. de Q.; Teixeira, M. M. \& da Silva, T. A. Role of CCR2 in orthodontic tooth movement. Am. J. Orthod. Dentofacial Orthop., 141(2):153-60, 2012.

Teixeira, C. C.; Khoo, E.; Tran, J.; Chartres, I.; Liu, Y.; Thant, L. M.; Khabensky, I.; Gart, L. P.; Cisneros, G. \& Alikhani, M. Cytokine expression and accelerated tooth movement. J. Dent. Res., 89(10):1135-41, 2010.

Dirección para correspondencia:

Prof. Paulo Sandoval Vidal

Departmento de Odontopediatria y Ortodoncia

Facultad de Odontología

Universidad de La Frontera

Manuel Montt \#115

Temuco

CHILE

Email: paulo.sandoval@ufrontera.cl

Recibido : 01-12-2018

Aceptado: 08-02-2019 